\title{
Inertial migration of rigid spherical particles in Poiseuille flow
}

\author{
By JEAN-PHILIPPE MATAS ${ }^{1}$, JEFFREY F. MORRIS \\ AND ÉLISABETH GUAZZELLI ${ }^{1}$ \\ ${ }^{1}$ IUSTI - CNRS UMR 6595, Polytech' Marseille, Technopôle de Château-Gombert, \\ 13453 Marseille cedex 13, France \\ ${ }^{2}$ Halliburton, 3000 N. Sam Houston Parkway East, Houston, TX 77032, USA
}

\begin{abstract}
An experimental study of the migration of dilute suspensions of particles in Poiseuille flow at Reynolds numbers $R e=67-1700$ was performed, with a few experiments performed at $R e$ up to 2400 . The particles used in the majority of the experiments were neutrally buoyant spheres with diameters $d$ yielding a ratio of pipe to particle diameter in the range $D / d=8-42$. The volume fraction of solids was less than $1 \%$ in all cases studied. The results of G. Segré \& A. Silberberg (J. Fluid Mech. 14, 136, 1962) have been extended to show that the tubular pinch effect in which particles accumulate on a narrow annulus is moved toward the wall as $R e$ increases. A careful comparison with asymptotic theory for Poiseuille flow in a channel was performed. Another inner annulus closer to the centre, and not predicted by this asymptotic theory, was observed at elevated $R e$. As $R e$ is increased, the distribution of particles over the cross-section of the tube at the measurement location, lying at a distance $L \doteq 310 D$ from the entrance, changes from one centred at the annulus predicted by the theory to one with the particles primarily on the inner annulus. The case of slightly non-neutrally buoyant particles was also investigated. A particle trajectory simulation based on asymptotic theory was performed to facilitate the comparison of theory and the experimental observations.
\end{abstract}

\section{Introduction}

A rigid sphere immersed in a spatially varying shear flow will undergo a lateral, or cross-stream, motion in the presence of inertia. Inertia is necessary to break the linearity of Stokes equations, under which lateral migration is forbidden, as demonstrated by Bretherton (1962). In a study of the Poiseuille flow of a dilute suspension of neutrally buoyant spheres, Segré \& Silberberg (1962) observed that a single rigid sphere in pipe flow migrated to an equilibrium position with its centre located at $r=0.6 R, R$ being the pipe radius. The phenomenon of radial migration driven by inertia was termed the tubular pinch effect to indicate that the uniform distribution of particles over the pipe cross-section converges, or is 'pinched', to a narrow annulus as the suspension moves downstream. These experiments prompted a strong interest in the suspension community, because at the time of the observations there was no theoretical explanation of this experimental result. A series of experiments followed, which investigated lateral forces on a sphere in several flow configurations: migration in Poiseuille flow in the absence of particle rotation (Oliver 1962), for non-neutrally buoyant spheres in vertical flows (Repetti \& Leonard 
1964; Jeffrey \& Pearson 1965; Karnis, Goldsmith \& Mason 1966; Aoki, Kurosaki \& Anzai 1979) and plane Poiseuille flow (Tachibana 1973). These experiments, beyond confirming Segré \& Silberberg's observations, showed in particular that the equilibrium position was shifted towards the axis when particles were lagging the flow, and towards the wall when they were leading it. Experiments were performed for pipe-scale Reynolds numbers $R e=\bar{U} D / v$ larger than 30 (where $\bar{U}$ is the mean axial velocity, $D=2 R$ and $v$ is the kinematic viscosity of the fluid). These experiments indicated that the equilibrium position lies closer to the wall in the presence of increased inertia. While inertially driven migration can be explained by consideration of a single particle, Han et al. (1999) confirmed that it was a very robust phenomenon which could be observed for volume fractions up to $\phi=0.2$.

The only theoretical evidence supporting lateral migration of a single rigid sphere at the time of the experiments of Segre \& Silberberg was that of Rubinow \& Keller (1961) who calculated the Magnus effect for a rigid sphere in a uniform flow. This force, always directed towards the pipe centreline, could not predict the existence of an equilibrium away from the axis. By a matched asymptotic expansion calculation, Saffman (1965) demonstrated that a rigid sphere in a linear shear flow experienced a lateral force proportional to its slip velocity relative to the fluid streamline going through its centre. The departure from $r=0.6 R$ observed for non-neutrally buoyant spheres in vertical flow, toward the wall for particles which lead the flow, and toward the axis for particles which lag the flow (Jeffrey \& Pearson 1965), suggested that Saffman's lift played a major role in the migration of non-neutrally buoyant spheres. Ho \& Leal (1974) calculated the force exerted on a rigid particle in a quadratic bounded shear flow in the case of small Reynolds numbers by a regular perturbation method, and were able to show that variation of the shear rate combined with the presence of a wall acting to create a repulsion resulted in an equilibrium position at $r=0.6 R$ consistent with the experimental results of Segré \& Silberberg. Schonberg \& Hinch (1989) succeeded in lifting the low-Reynolds-number restriction by integrating the solution of the differential equations yielded by the matched asymptotic expansion method, and predicted the evolution of the equilibrium position for Re up to 150 . Hogg (1994) applied the same method to the problem of non-neutrally buoyant particles in Poiseuille flow. Asmolov (1999) extended this method to Reynolds numbers as large as 1500 by the use of an orthonormalization method to integrate the equations of the matched asymptotic expansion problem. Note that, in all of the asymptotic theory, the particle scale Reynolds number satisfies $\operatorname{Re}_{p} \ll 1$ where $\operatorname{Re}_{p} \equiv \operatorname{Re}(d / D)^{2}$, with $d$ the diameter of the particles and $D$ that of the pipe.

To date there has been little experimental study examining the validity of these theories at elevated $R e$. The objective of the present work is to study the influence of inertia on the radial migration of rigid neutrally buoyant spheres in Poiseuille flow. We extended the Segré-Silberberg experiments up to $R e=1700$ and considered particle sizes yielding $D / d=8-42$; a few experiments at larger $R e$ will also be described. The equilibrium radial position was examined and is termed the Segré-Silberberg annulus. We also found a novel feature of inertial migration in the form of an inner annulus and we examine whether it is another stable equilibrium position. Since the data were collected at a single point at the end of the pipe, we did not study the evolution of the concentration profile with position and therefore we did not check that the distribution having an inner annulus reached a steady state within the length of the tube. However, some observations such as the fact that the distribution switches from one centred at the Segré-Silberberg position to one centred on the inner annulus when $R e$ is increased and that this occurs at the same $R e$ for all particles provide 


$\begin{array}{crrrrrr}d(\mu \mathrm{m}) & 190 \pm 10 & 450 \pm 50 & 550 \pm 50 & 750 \pm 500 & 900 \pm 50 & 1000 \pm 50 \\ D / d & 42 \pm 2.5 & 17 \pm 2.5 & 15 \pm 1.5 & 10.5 \pm 1 & 9 \pm 0.5 & 8 \pm 0.5\end{array}$

TABle 1. Particle diameters and pipe to particle diameter ratios.

The pipe inner diameter is $D=8 \mathrm{~mm}$.

alternative arguments in favour of the stability of the inner annulus that will be discussed in detail. We have also examined the effect of a small buoyancy on the migration process.

We first describe in $\S 2$ the experimental techniques and the method used for measurements of the particle distribution. The experimental results are presented in $\S 3$. After reviewing the analysis and results of the matched asymptotic expansion method in $\S 4$, we compare and discuss the predictions of this theory with our experimental results in $\S 5$.

\section{Experimental techniques}

\subsection{Particles and fluid}

We used as particles polystyrene beads with diameters in the range $d=190 \mu \mathrm{m}-$ $1 \mathrm{~mm}$, as indicated in table 1. The particles were supplied by Maxi-Blast (South Bend, IN, USA) and were found to be spherical. However, the pump which circulated the suspension tended to flatten a small fraction of the larger beads with each circulation through the apparatus. Therefore after some time, some of the particles were no longer perfectly spherical. These non-spherical particles were observed to have a different migration behaviour from the spheres and this was found to be a significant source of scatter in the data for the large particles. The density of the suspending fluid $\rho_{f}$ was matched to the density of the particles $\rho_{p}$ by using a mixture of glycerol and water. The density of the fluid was measured using a hydrometer, or float densimeter, from ERTCO (West Paterson, NJ, USA). The particle density was determined by finding a fluid of measured density in which no sedimentation of a batch of particles occurred. The densities of the different particle sets were in the range $\rho_{p}=1.049-1.053 \mathrm{~g} \mathrm{~cm}^{-3}$, for which the suspending fluid composition in glycerol : water fraction was between $0.21: 0.79$ and $0.23: 0.77$ at a temperature $T=25^{\circ} \mathrm{C}$. The density of the particles among a given set was homogeneous, except for the $D / d=17$ and $D / d=15$ sets, for which slightly more and less dense particles were observed simultaneously at low flow rates, and thus were used to study the influence of a dispersion in density upon particle distribution. The suspension was maintained at $T=25^{\circ} \mathrm{C}$, a temperature for which the viscosity of the suspending fluid was in the range $\eta=1.45-1.55 \pm 0.03 \mathrm{cP}$.

\subsection{Experimental apparatus}

The experimental apparatus test section was a horizontal glass tube of inner diameter $D=8 \mathrm{~mm}$. The tube had a length $L=2.6 \mathrm{~m}$, longer than the entry length $L_{e} \sim 50 \mathrm{~cm}$ necessary for the laminar flow to fully develop at $R e \approx 2000$; above this $R e$, the laminar flow becomes unstable. The measurement of particle distribution was made at a position $2.5 \mathrm{~m}$ from the entrance. In order to ensure that the flow in the pipe was undisturbed by perturbations from a pump, the flow was driven by gravity. The suspension was delivered to the tube by overflow from a tank positioned at a fixed height to an outlet of variable height, passing through the glass tube as indicated in 


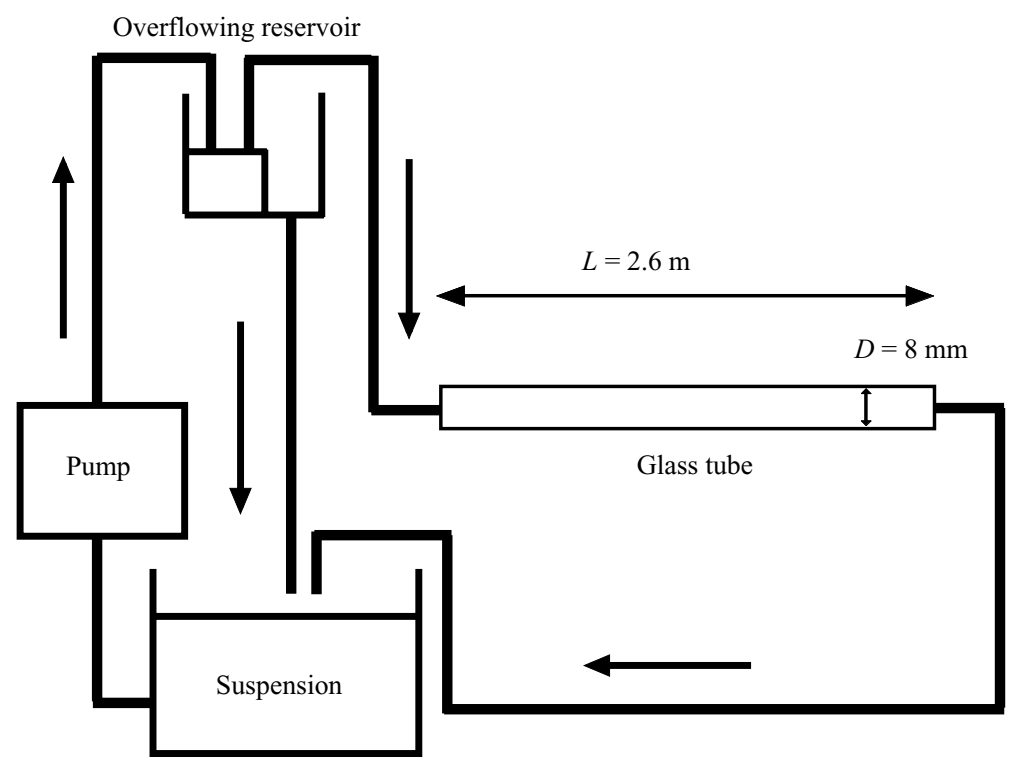

FIGURE 1. Diagram of the experimental apparatus used. The diameter noted on the figure, $D=8 \mathrm{~mm}$, is the inner diameter of the glass tube. The overflowing reservoir is elevated relative to the remainder of the apparatus, with the flow rate controlled by the elevation of the overflowing reservoir above the thermostated reservoir.

figure 1. A Moineau progressing cavity pump (PCM model MR2.6H24) carried the suspension from a lower thermostated reservoir back to the overflowing tank. The pump was, however, isolated from the flow through the glass tube. The flow rate $Q$ was determined by measuring a collected volume of the suspension at the outlet of the tube in a given time.

\subsection{Measurement of particle position}

The position of particles in the pipe was measured by making a vertical section of the tube with a laser sheet and recording the position of the particles intersecting the sheet with a camera. The position was measured at a fixed location corresponding to $L / D \doteq 310$ from the entrance of the tube. To limit the deformation of the image caused by refraction effects, a Plexiglas vessel was placed around the test section of the tube and filled with glycerol. The index of refraction of glycerol $n_{G}=1.48$ is close to the index of refraction of glass. The air-glass refraction at the cylindrical outer wall of the tube was then transferred to an air-glass refraction at the plane wall of the Plexiglas box (see figure 2). There is also a deformation of the image associated with the refraction at the inner wall of the tube. However, the index of the glycerol-water mixture is $n_{F}=1.360 \pm 0.002$ at $T=25^{\circ} \mathrm{C}$ (measured with an Abbe refractometer), rather close to the index of glass and thus this effect causes only limited deformation.

The images were obtained using a digital video camera at 10 images per second. The frame rate is not increased beyond this value in order to avoid any particle being present in two consecutive images and being counted twice. The laser sheet being filmed under an angle of $40^{\circ}$, is a three-dimensional object and we have to ensure that its depth is smaller than the field depth in our optical conditions: the aperture chosen is therefore the largest available with this camera, i.e. an aperture number of 11. This aperture allows a good focus over the whole width of the laser sheet. The shutter speed is fixed at an opening period of $1 / 1600 \mathrm{~s}$. The images are then 


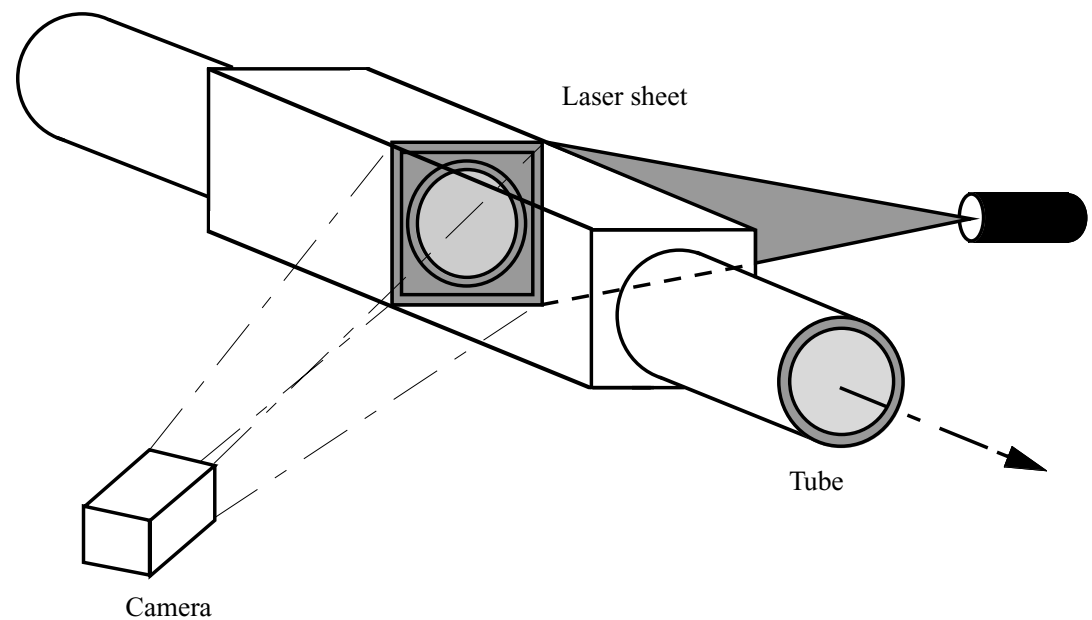

FIGURE 2. Experimental measurement of the particle positions: the camera captures images of those particles intersecting the laser sheet. The air-glass refraction at the cylindrical outer wall of the glass tube is transformed into a plane wall refraction (see figure 3).
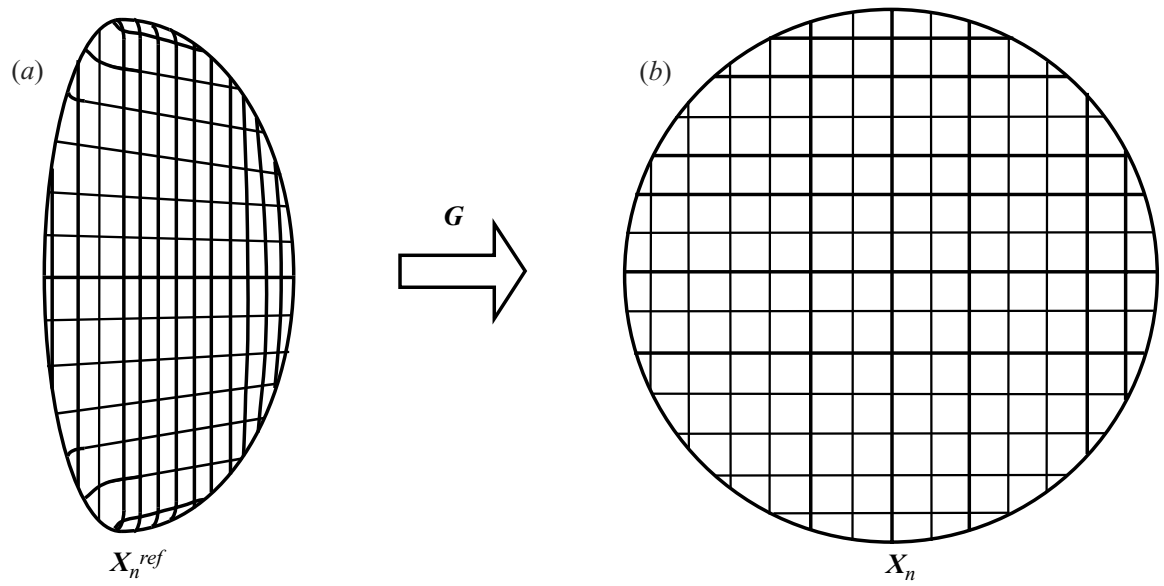

Figure 3. (a) Transformation of the observed refracted calibration grid to $(b)$ its actual symmetric form.

analysed with the public domain image processing software NIH Image to yield the position of the intersected particles. Each image is thresholded to turn the particles present on the section into separate homogeneous white zones. The counting of the particles is achieved by keeping on each thresholded image only those white zones the area of which lies between a minimum and a maximum value: these values are chosen in order to avoid the counting of any particle of a different size which could have remained in the system. This procedure yields the position $\boldsymbol{X}_{p}^{r e f}$ of each particle centre on the refracted section. These data are then exported to Matlab where a calibration is applied to restore the symmetry of the image. In order to do this calibration, a disk having the same diameter as the glass tube and bearing an array of regularly spaced points $\boldsymbol{X}_{n}$ has been inserted into the tube. An image of this grid after refraction was recorded under the same optical conditions used in particle position measurements, and the coordinates $\boldsymbol{X}_{n}^{\text {ref }}$ of its points in refracted space were 

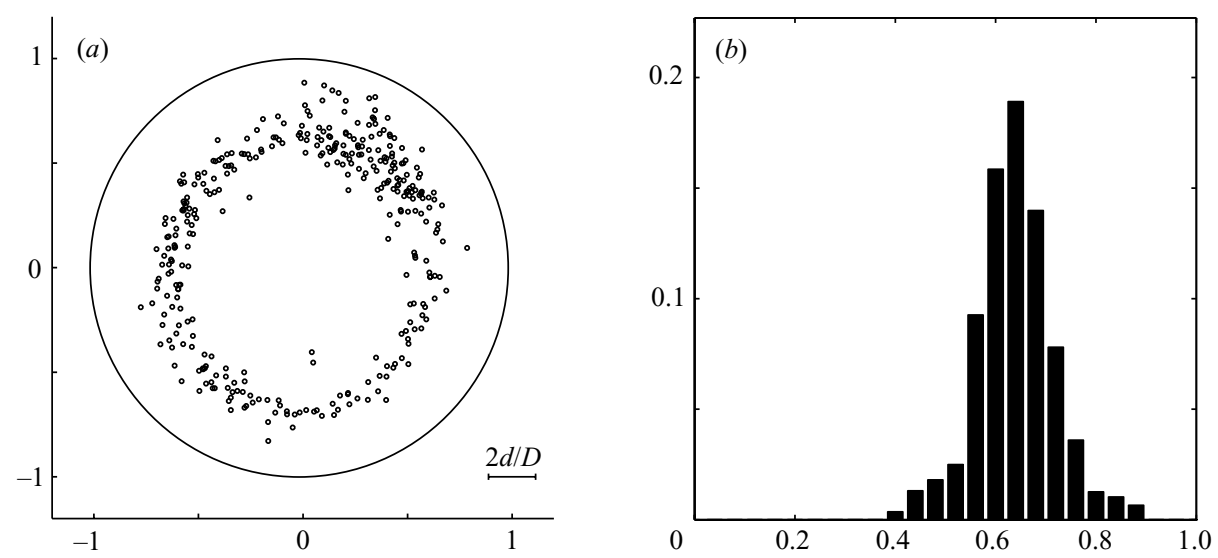

Figure 4. (a) Particle distribution over a cross-section and $(b)$ the corresponding histogram showing the probability $p(r)$ as a function of the dimensionless radius, for $R e=67$ and $D / d=9$. In $(a)$, both axes are labelled with lengths scaled by the tube radius, and the bar at the lower right-hand side shows the mean particle diameter on the same scale.

measured. This gives the value of the inverse refraction function $\boldsymbol{G}$ at the refracted grid points: $\boldsymbol{X}_{n}=\boldsymbol{G}\left(\boldsymbol{X}_{n}^{r e f}\right)$. The measured particle positions $\boldsymbol{X}_{p}^{r e f}$ are mapped onto the refracted grid $\boldsymbol{X}_{n}^{r e f}$ : the position $\boldsymbol{X}_{p}$ of the particles in non-refracted space is then simply given by an interpolation of the function $\boldsymbol{G}$ at the refracted particle positions $\boldsymbol{X}_{p}^{r e f}$.

The concentration profiles were measured for very dilute suspensions. The particle volume fraction $\phi$ had to be small enough to ensure that particles were far apart, so we always performed experiments for $\phi<0.01$. Thus, interparticle interactions are argued to be of minor influence. The laser sheet was positioned at $L / D=310$, at the end of the tube (we assume a random distribution at the tube entrance). The particle positions were then measured over at least 5000 images for each flow condition to yield statistically significant results.

\section{Experimental results}

Measurements of the particle distribution over a cross-section have been performed for each of the sets of particles of table 1. We first present the influence of the Reynolds number and particle size on particle distribution for neutrally buoyant particles, and then for the case of particles slightly different in density from the fluid.

\subsection{Inertial migration of neutrally buoyant particles}

The mixture of glycerol and water chosen to ensure that particles are neutrally buoyant has a relatively low viscosity, with $\eta$ between 1.45 and $1.55 \mathrm{cP}$ depending on the set of particles used. At the lowest steady flow rate, this gives a lower limit of $R e=60$. The majority of experiments were carried out from near this lower limit to $R e \approx 1700$, close to the transition between laminar and intermittent flows, with a few experiments performed at higher Re. Figure 4(a) shows the distribution of $D / d=9$ particles over a cross-section of the flow for $R e=67$ and figure 5(a) shows the result of a similar measurement performed for $R e=350$. Note that the scaling of 

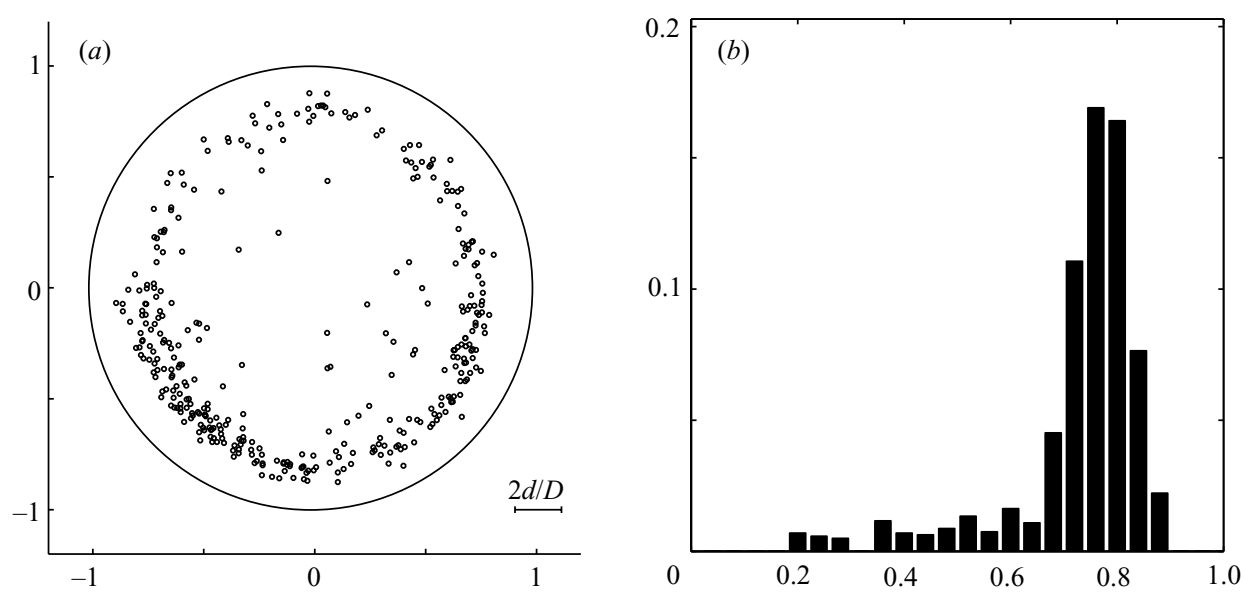

Figure 5. (a) Particle distribution over a cross-section and $(b)$ the corresponding histogram showing the probability $p(r)$ as a function of the dimensionless radius, for $R e=350$ and $D / d=9$. In $(a)$, both axes are labelled with lengths scaled by the tube radius, and the bar at the lower right-hand side shows the mean particle diameter on the same scale.

length in these figures is with the inner radius so that the pipe wall is at 1 . These results represent the cumulated particle positions over the whole experiment. The bulk concentration is $\phi=5 \times 10^{-3}$.

A comparison of figures $4(a)$ and 5(a) shows that the equilibrium position is shifted towards the wall when the Reynolds number is increased. To make such information more precise, we characterize the radial distribution by a radial probability function. We begin by building a histogram from all the measured radial positions $r$ on the cross-section. This histogram, when normalized, yields the probability density function (p.d.f.) $p_{r}(r)$ associated with the probability of a particle being at a radius $r$. A preferred radial position due to migration corresponds to a maximum of the p.d.f. $p(r, \theta)$, associated with the probability of being at a radius $r$ and a given angular position $\theta$. The experimentally measured $p_{r}$ is related to $p$ by $p_{r}(r)=$ $\pi^{-1} \int_{0}^{2 \pi} p(r, \theta) r \mathrm{~d} \theta$. Assuming that $p$ depends only upon $r$, this yields $p(r)=p_{r}(r) / 2 r$. The histograms showing the p.d.f. $p(r)$ extracted from figures $4(a)$ and $5(a)$ are shown on figures $4(b)$ and $5(b)$, respectively. The radius of equilibrium, i.e. the SegréSilberberg equilibrium position, is then taken to be the position, $r_{\max }$, corresponding to the maximum of the histogram. To allow a greater precision, we take the value of the maximum of the parabolic curve passing through the maximum and its two neighbouring values. For the $D / d=17$ and $D / d=15$ particles, the density among a given set was not homogeneous, causing a fraction of the particles to migrate towards the bottom or towards the top of the cross-section. For these particles, the SegreSilberberg equilibrium position was determined by considering only the particles located in a horizontal slice through the section centre.

Several sources of uncertainty can account for the dispersion observed around the equilibrium position on figures 4 and 5 . The measured centre of each particle in these figures corresponds in fact to the centre of mass of the particle intersection by the laser sheet; the difference between this position and the effective particle centre can therefore be of the order of one particle size. As indicated by the horizontal bars in the lower right-hand sides of figures $4(a)$ and $5(a)$, this can result in a significant error 
for the larger particles. Errors can also occur during the calibration: the operation applied on the refracted images to suppress refraction effects is difficult for particles close to the top and bottom of the pipe, where refraction is maximum. This imperfect calibration will result in a broadening of the histogram of radial positions. The dispersion in size observed among a given set of particles (see table 1), and the nonsphericity of some particles can also contribute to the dispersion observed around the equilibrium position.

When the Reynolds number is further increased, the equilibrium position is shifted still closer to the wall. However, a second annulus appears on the cross-section at smaller $r$ as can be seen in figure 6(a). This inner annulus was seen for $R e>600$ for $D / d=9-17$, and for $R e>1200$ when $D / d=42$. It is much broader than the SegréSilberberg annulus (which is closer to the wall), and appears centred on $r=0.5 R$ for most of our data, spreading from $r / R=0.3$ to $r / R=0.7$. This distribution is thus less sharply peaked than the regular Segré-Silberberg equilibrium position. When the maximum of $p(r)$ corresponds to the position of this inner annulus, we choose to take the position of the local maximum of $p(r)$ at larger $r$ to be the SegréSilberberg annulus, plotted on figure 9. For Reynolds numbers close to the transition to intermittency, only this inner annulus can be clearly observed (see figure 6(c) for results at $R e=1650$ and $D / d=17$ ). For these Reynolds numbers, there are generally very few particles remaining on the Segré-Silberberg annulus. It is then difficult to estimate the equilibrium position located near the wall for these $R e$. It is also surprising to observe that the inner annulus is robust enough to survive even into the intermittent regime at $R e \approx 2400$ (see figure $6 e$ ). It is worth mentioning that the transition to intermittency is moved to lower Reynolds numbers even by a small fraction of large particles as shown by Matas, Morris \& Guazzelli 2003.

To complete the description, we can see that, as $R e$ is increased, the highest probability of finding a particle switches from the outer (Segré-Silberberg) annulus to the inner annulus. This is clearly seen in figure 7 where are plotted the probabilities of finding a particle on the inner, $P_{i n}$, or outer, $P_{\text {out }}$, annulus as a function of Reynolds number for different particle sizes. The smallest $D / d=42$ particles, for which the inner annulus is only oserved for $R e>1200$, are not represented. The arbitrary location of the inner annulus is $0.22 \leqslant r / R \leqslant 0.78$ and that of the outer annulus is $0.78<r / R \leqslant 0.98$. The behaviour is remarkably similar as far as the independence on particle size is concerned. We observe that $P_{\text {in }}$ becomes larger than $P_{\text {out }}$ for $R e \approx$ 650.

For the smallest particles used in this study $(D / d=42)$, the behaviour is somewhat different. The entry length necessary to achieve radial migration seems to be larger than the length of our glass tube (see $\S 5$ for the discussion of this entry length). A typical concentration profile and the corresponding histogram showing $p(r)$ for these particles at $R e=780$ are shown in figure 8 . Though there are particles present over almost the entire cross-section, the outer circle can be clearly identified in the histogram, allowing measurement of the outer circle position. However, for low Re, this outer circle does not appear clearly enough in the histogram, and measurements were then only carried out for $R e>200$ for these particles. As a side but important comment, the fact that the distribution on figure 8 has a uniform background other than the accumulation on the Segré-Silberberg annulus gives confidence that the initial distribution at the pipe entrance is uniform.

We present in figure 9 the variation of the measured Segré-Silberberg equilibrium position (the outer annulus position) with the Reynolds number for each set of 

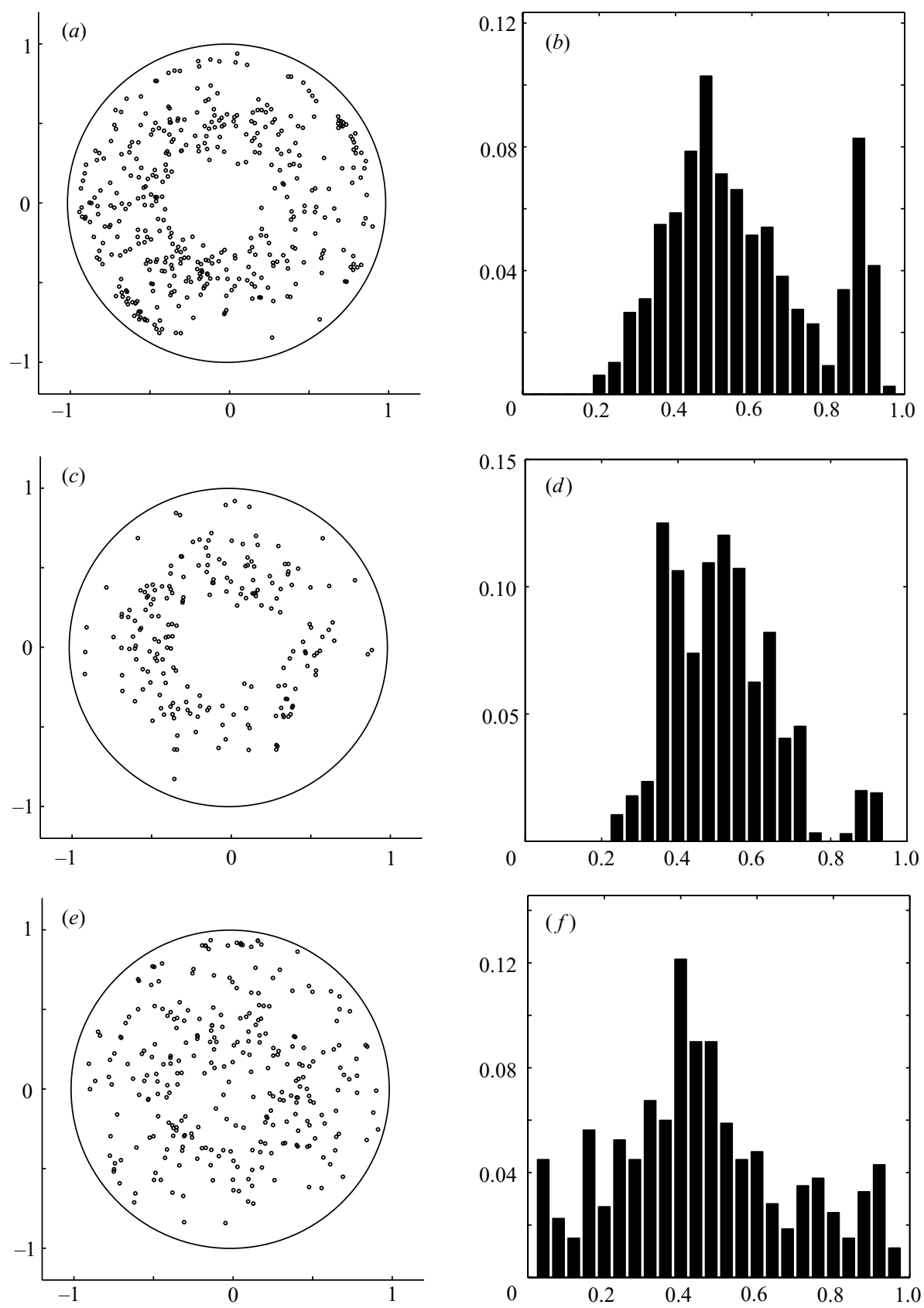

FiguRE 6. Particle distributions over a cross-section for $D / d=17$ at (a) $R e=1000$, (c) $R e=1650$ and $(e) R e=2400$ and the respective histograms $(b),(d)$, and $(f)$ showing the probability $p(r)$ as a function of the dimensionless radius. In $(a),(c)$ and $(e)$, both axes are labelled with lengths scaled by the tube radius. Note that at $R e=2400$ the flow is intermittent.

particles. The error in $R e$ results from the uncertainty in the viscosity $\eta$, and was $\Delta R e= \pm 0.02 R e$. The error in the radius results from actual broadness in the maximum and calibration errors. It was larger at small Reynolds numbers than at large $R e$, as 


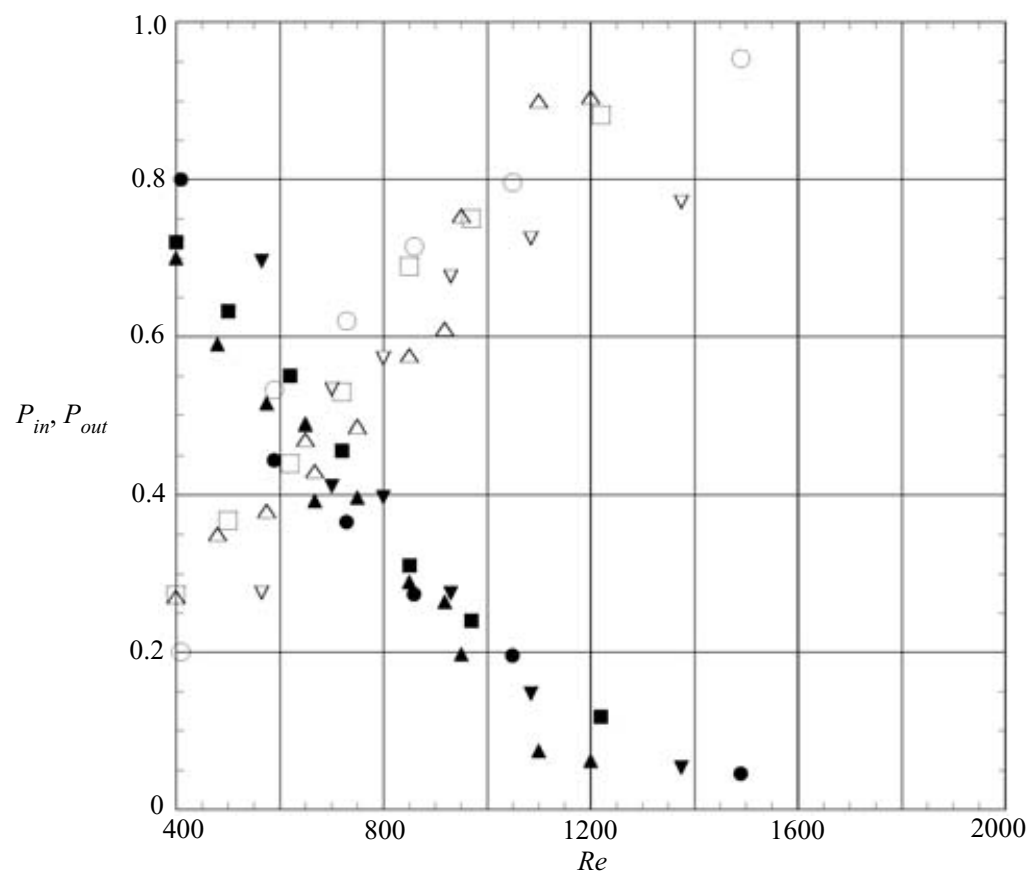

FIgURE 7. Probabilities of finding a particle on the inner, $P_{i n}$ (open symbols), or outer, $P_{\text {out }}$ (solid symbols), annulus as a function of Reynolds number for different particle sizes: $\bigcirc, D / d=17 ; \square, 15 ; \triangle, 10.5 ; \nabla, 9$.
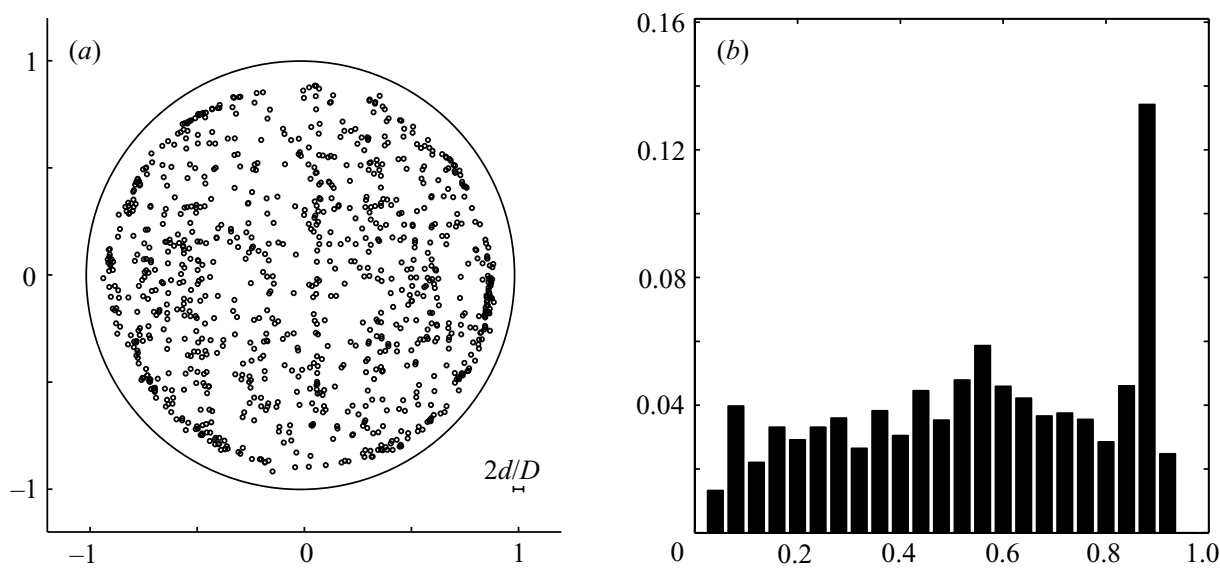

FIgURE 8. (a) Particle distribution over a cross-section and $(b)$ the corresponding histogram showing the probability $p(r)$ as a function of the dimensionless radius for $R e=780$ and $D / d=42$. In $(a)$, both axes are labelled with lengths scaled by the tube radius, and the bar at the lower right-hand side shows the mean particle diameter on the same scale.

indicated on figure 9 for three typical data points. The solid line is the prediction of the asymptotic theory which will be discussed in the next section. Figure 10 shows an expanded view of these results for small $R e$. We will discuss in $\S 4$ how these results compare with theoretical predictions. 


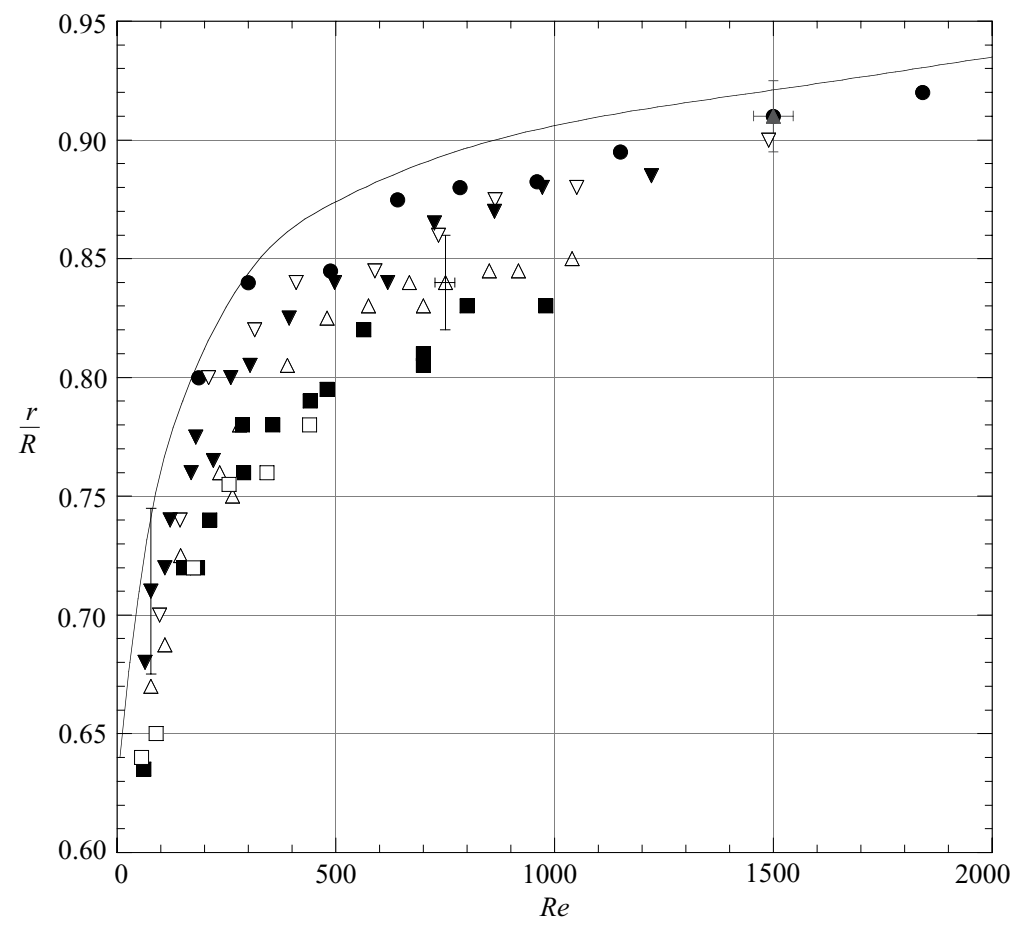

FIGURE 9. Segré-Silberberg equilibrium position of a rigid spherical particle as a function of Reynolds number for different particle sizes: $\bullet, D / d=42 ; \nabla, 17 ; \nabla, 15 ; \Delta, 10.5 ; \mathbf{\square}, 9 ; \square, 8$. The solid curve represents the prediction of the asymptotic theory.

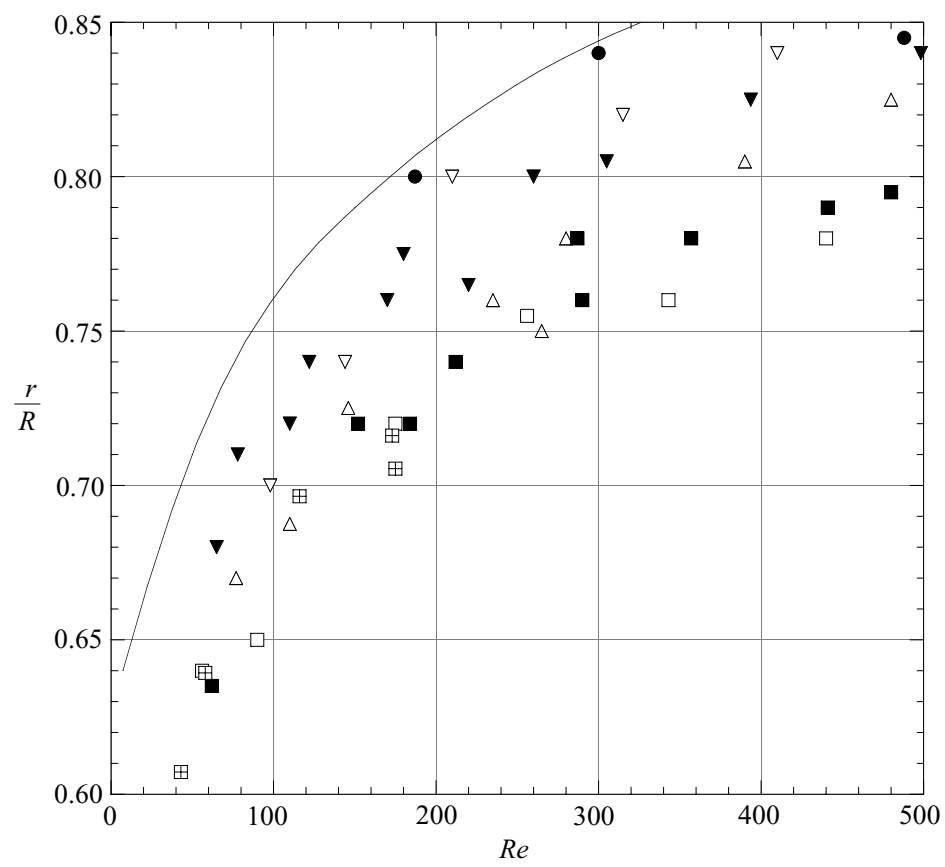

Figure 10. Enlargement of part of figure $9 . \boxplus$, Segré \& Silberberg (1962) data $D / d=9$. 

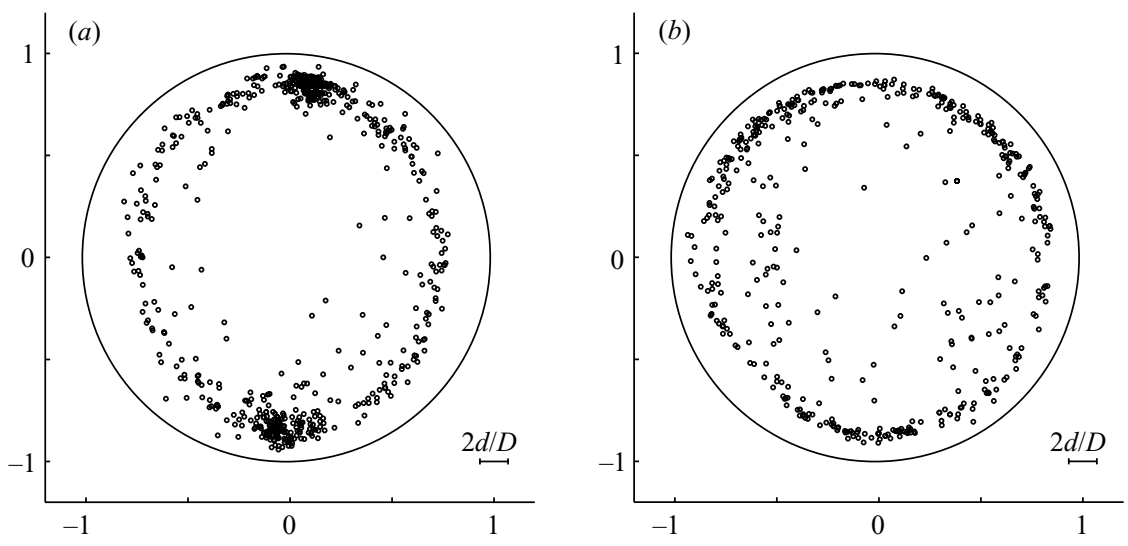

FIGURE 11. Particle distribution over a cross-section at $(a) R e=170$ and $(b) R e=390$, for a set of particles of $D / d=15$ having a dispersion $\Delta \rho$ about a mean density $\bar{\rho}_{p}=\rho_{f}$. The axes are labelled with the dimensionless radius, $r / R$, and the bar represents the particle diameter on this scale.

In order to compare our results to the results of Segré \& Silberberg (1962) for large Reynolds numbers, we consider their results for $R e=43-175$, obtained with particles having $D / d=9$. Table 1 of their paper does not explicitly give the maximum of the histogram for their measurements, but gives instead $r_{\text {lim }}$ and $\bar{r}$, respectively, the outer radial position where the concentration goes to zero and the mean radial position. The value $r_{l i m}$ overestimates the equilibrium position, while the value $\bar{r}$ certainly underestimates it since the skewness of the histograms appears to be negative. However, the histograms of the concentration profiles obtained far from the entrance (after a length $L=120 \mathrm{~cm}$ ) in Segré \& Silberberg (1962) are very sharp. Hence, we assume that $\bar{r}$ is close to the maximum for these points. We have plotted the position $\bar{r} / R$ of the corresponding experiments in figure 10, from which it can be seen that our results for large particles $(D / d=9)$ are in good agreement. It should also be mentioned that while Segré \& Silberberg made some measurements at $R e=694$, they do not provide the value of $\bar{r}$, but only $r_{\text {lim }} / R=0.80$. This latter value is in good agreement with the present data for $D / d=9$ of figure 9 .

The results of figures 9 and 10 clearly show that the Segré \& Silberberg equilibrium position moves toward the wall as the Reynolds number is increased, in agreement with the theory for point particles. At a given $R e$, our experiments demonstrate that this equilibrium position is shifted toward the centre as $D / d$ is decreased, i.e. as the particle size increases for a given pipe diameter. This size effect is magnified for large $R e$.

\subsection{Influence of a slight sedimentation on the inertial migration}

As mentioned in $\S 2$, in the case of the $D / d=15$ particles, a slight dispersion in density of $\rho_{p}=1.052 \pm 0.001 \mathrm{~g} \mathrm{~cm}^{-3}$ had an influence at small flow rates. At $R e=170$, light and heavy particles tend to migrate to equilibrium positions shifted closer to the top and bottom of the cross-section, respectively. The neutrally buoyant fraction of the particles remains on the Segré-Silberberg annulus (see figure 11a). The resulting distribution is slightly deformed, its height being larger than its width. A measure of buoyancy is given by the dimensionless number $B=U_{S} / \bar{U}=2 / 9 a^{2} \Delta \rho g / \eta \bar{U}$, where $\bar{U}=U_{m} / 2$ is the average axial velocity and $U_{S}$ is the Stokes' sedimentation velocity. 
(a)

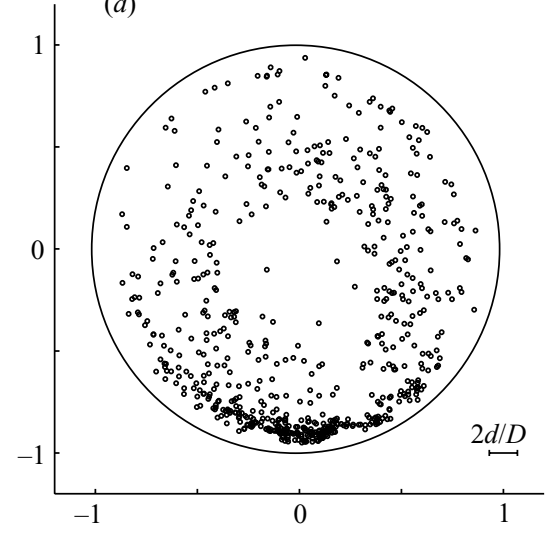

(b)

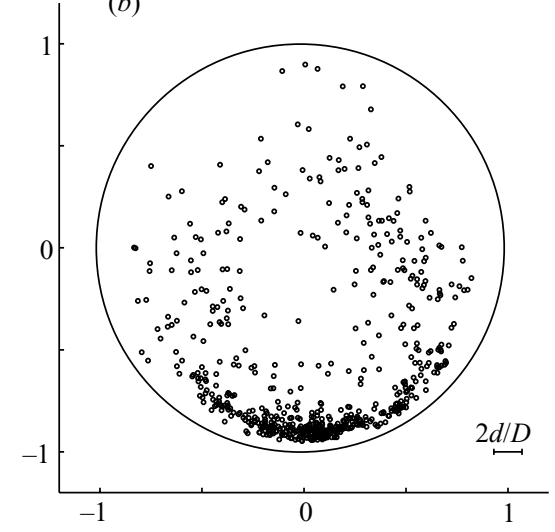

FIGURE 12. Particle distribution over a cross-section at $R e=650$ in the presence of slight sedimentation: $(a) B=1.5 \times 10^{-3}$ and $(b) B=2.5 \times 10^{-3}$ for $D / d=15$. The axes are labelled with the dimensionless radius, $r / R$, and the bar represents the particle diameter on this scale.

For these $D / d=15$ particles, we estimate the maximum $B_{\max } \approx 0.0036$ at $R e=170$ (figure $11 a$ ) and $B_{\text {max }} \approx 0.0016$ at $R e=390$ (figure $11 b$ ). When the flow rate is increased, it can be seen on figure $11(b)$ that the lower and upper equilibrium positions are now on the Segré-Silberberg annulus. Also the radius of the annulus has been slightly increased. This seems to indicate that the radial inertial force is dominated by buoyancy for $R e=170$ while it is stronger than buoyancy for $R e=390$.

Measurements were also made for particles all slightly denser than the fluid. Note that these particles also have a slight dispersion in density. If the pipe were long enough, the slightly heavier particles are predicted by the theory, see $\S 4$, to reach a single equilibrium position close to the bottom of the pipe. In fact, because the buoyancy force is weak, the development length for settling is long relative to that for inertial migration alone. Thus, we measure here the particle distribution in the developing regime, when the force responsible for radial migration is not negligible compared to gravity. Figure $12(a)$ shows the particle distribution at the end of the tube for a density difference $\Delta \rho=1.5 \times 10^{-3} \mathrm{~g} \mathrm{~cm}^{-3}(B=0.0015)$ and $R e=650$ in the case of the $D / d=15$ particles. The observed patterns noted in the previous subsection remain clearly recognizable. Particles are present on the outer circle corresponding to the Segré-Silberberg equilibrium position, though mainly on its lower part, and particles are observed on an inner annulus of radius $r \sim 0.5 R$. Particles appear to sediment along directions imposed by the radial migration. When sedimentation is further increased, as in figure $12(b)$ where $\Delta \rho=2.5 \times 10^{-3} \mathrm{~g} \mathrm{~cm}^{-3}(B=0.0025)$, particles at the end of the tube are mainly present at the bottom of the pipe but also on the upper part of the inner annulus. The entire pattern is shifted toward the bottom of the pipe at this larger value of $B$.

\section{Asymptotic theory}

We now describe the method of matched asymptotic expansions which has been developed by Schonberg \& Hinch (1989), Hogg (1994) and Asmolov (1999), to evaluate the lift force on a spherical rigid particle in a two-dimensional Poiseuille flow. We consider a Poiseuille flow between two infinite parallel plates separated by a distance $l$, which is disturbed by a rigid sphere of radius $a=d / 2$ (see figure 13); the radius is specifically denoted because of its use in theoretical developments of 


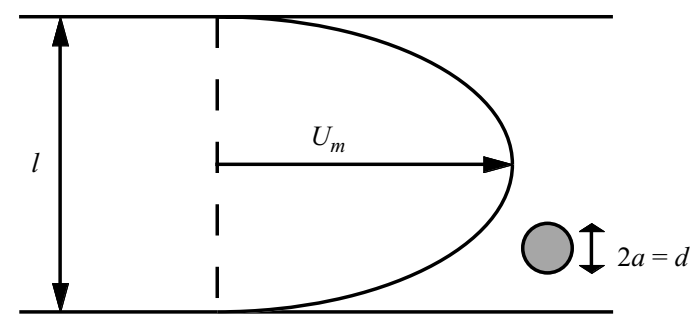

FIGURE 13. Basic parameters used in the description of channel flow.

other authors. The origin of the coordinate system is taken to be instantaneously at the centre of the sphere, thus moving with the sphere. The Reynolds number of the channel flow $R_{c}$ as defined by Asmolov differs slightly from our definition in using the maximum velocity in the channel $U_{m}$ rather than the mean: $R_{c}=U_{m} l / v$. Both the particle Reynolds number $R e_{p}=U_{m} d^{2} / 2 v l=R_{c}(a / l)^{2}$ and the size of the sphere relative to the channel width $\alpha=d / l$ are assumed to be small. In contrast, the channel Reynolds number $R_{c}$ is of order unity or larger. The basic asymptotic parameter in this problem can be defined as $\epsilon=R_{p}^{1 / 2}$, where $R_{p}=R e_{p} / 2$ is the particle scale Reynolds number used by Asmolov.

In the matched asymptotic expansion theory, two regions of the flow are considered. In the inner region close to the particle, the length scale is the particle radius $a=d / 2$. In the limit of small $\epsilon$, the leading-order governing equations reduce to the Stokes equations in an unbounded fluid. If the slip velocity of the particle relative to the fluid is small enough (in particular in the case of a neutrally buoyant particle), the leading-order solution is the stresslet velocity field. It corresponds to viscous flow driven by a symmetric force dipole (see Schonberg \& Hinch 1989; Asmolov 1999). If sedimentation is sufficiently strong, the leading-order solution is the Stokeslet (Hogg 1994; Asmolov 1999). In the outer region far from the particle, we must consider Oseen equations in which advection terms balance viscous terms. In this region, the new length scale is found by stretching the inner length scale by $\epsilon$. The solution to these new equations must then match the inner solution.

In the following, we consider neutrally buoyant particles. The matching condition can be encapsulated in the Oseen equation by introducing a singularity corresponding to the symmetric force dipole. The equation of the outer flow can then be reduced to a fourth-order ordinary differential equation for the Fourier transform of the lateral velocity, as shown, for instance, by equation (5.6) of Asmolov (1999). The lift force $F_{l}$ is just the Stokes drag $F_{S}=6 \pi \eta a V_{\text {migr }}$ associated to the lateral velocity $V_{\text {migr }}$. The differential equation is solved with the orthonormalization method used by Asmolov, ensuring a correct computation of the four independent solutions, as shown in the Appendix of Asmolov (1999). Using the numerical procedure described by Asmolov, we have computed the lift force for different Reynolds numbers, as displayed in figure 14. To allow an easier comparison with our experiments (see the following section), the force on figure 14 is plotted as a function of $2 z / l$ where $z$ is the distance from the centre of the channel. It is also convenient to scale this force by $R_{c}^{-1 / 2} \epsilon^{3}$. Close to the wall $(2 z / l$ close to one) the force is negative and pushes the particle towards the centre, whereas near the centre it is positive and pushes the particle towards the wall. The equilibrium position $z_{e q}$ thus corresponds to the zero of $F_{l}$. The equilibrium position as a function of $R e$ based upon the average velocity is plotted as the solid line on figures 9 and 10. It is important to note that for $R e>300$ the force profile exhibits a convex region of lower amplitude surrounded by two maxima (instead 


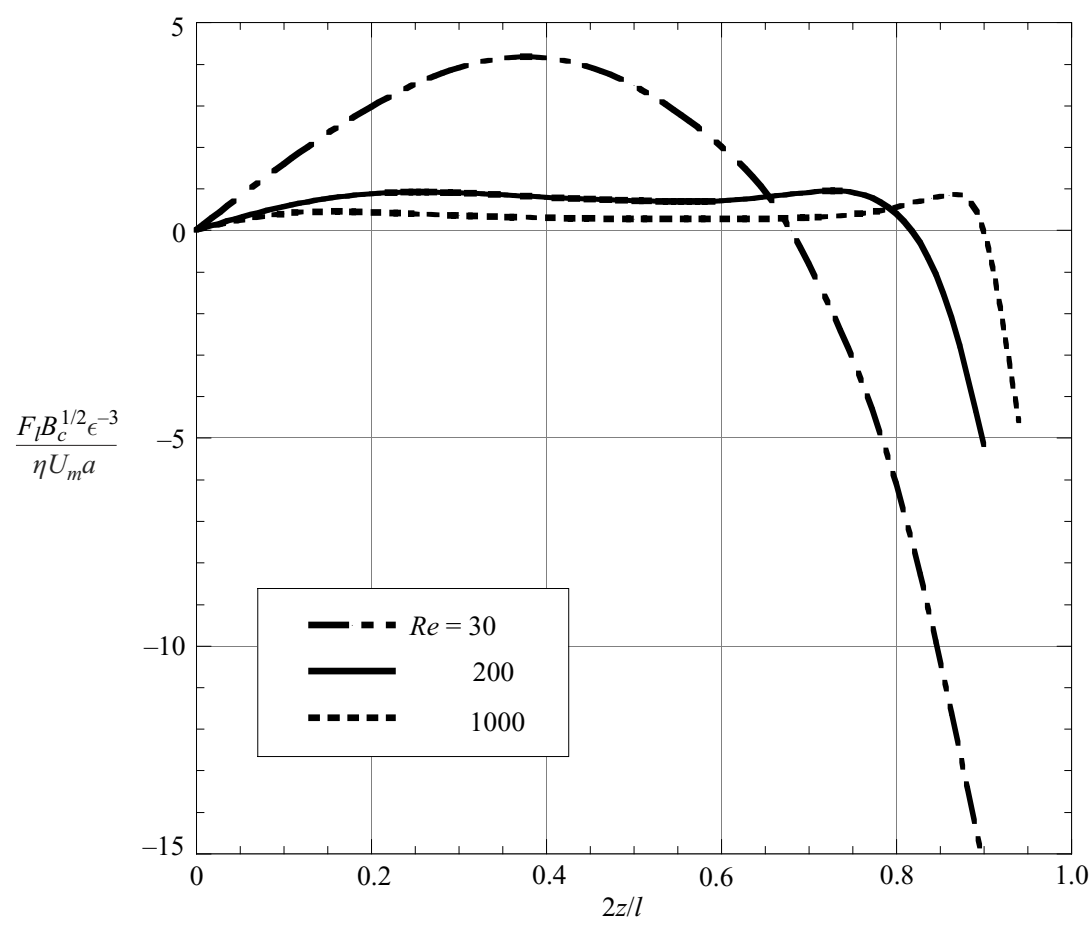

FIGURE 14. Variation of the lift force $F_{l}$ exerted on a rigid spherical particle in channel flow with position, calculated by the method of matched asymptotic expansions. The position $z=0$ corresponds to the channel centreplane.

of a single maximum) between the centre and the equilibrium position. Finally, for neutrally buoyant particles, the lateral motion of the particle can be described by the balance between the lift force and the Stokes drag associated to the lateral motion: $\boldsymbol{F}_{l}+\boldsymbol{F}_{S}=0$ where the drag force can be expressed as $\boldsymbol{F}_{S}=6 \pi \eta a \mathrm{~d} \boldsymbol{x} / \mathrm{d} t$ and $\boldsymbol{x}$ is the position in the cross-section; the motion along the flow is simply described by the Poiseuille velocity of the fluid at the particle centre. For the neutrally buoyant case, both forces are expected to be purely radial, but are written in vector form for later use.

In the case of slightly non-neutrally buoyant particles, we may safely continue to use the above estimate of the lift force as long as buoyancy meets two conditions. The first is that the stresslet velocity field dominates the Stokeslet velocity field for the matching condition. This has been stated by Hogg (1994) as $(a / l)^{-2} B / 2 \ll 1$, where $B$ is the buoyancy number defined previously as the ratio of the Stokes settling velocity to the channel average velocity $\bar{U}=U_{m} / 2$. The second condition is equivalent to inertia in the outer region being dominated by shear and not sedimentation, and can be stated as $R_{c}(B / 2)^{2} \ll 1$. Within these approximations, the lateral motion of the particle can be described by adding a sedimentation force to the force balance: $\boldsymbol{F}_{l}+\boldsymbol{F}_{S}+\boldsymbol{F}_{g}=0$ where $\boldsymbol{F}_{g}=4 \pi a^{3} \Delta \rho \boldsymbol{g} / 3$, where $\boldsymbol{g}$ is the acceleration due to gravity.

The entry length after which radial migration will have developed can be estimated from the magnitude of the radial force $F_{l}=A(d / l)^{4} \rho U^{2} l^{2} / 4$, by assuming that $F_{l}$ is balanced by a viscous Stokes drag $F_{S}=6 \pi \eta(d / 2) V_{\text {migr }}$. We have taken $A$ to be a typical magnitude of the scaled force. The results of figure 14 suggest that $A$ is a function of 


$$
\begin{array}{ccccccc}
D / d & 42 \pm 2.5 & 17 \pm 2.5 & 15 \pm 1.5 & 10.5 \pm 1 & 9 \pm 0.5 & 8 \pm 0.5 \\
\operatorname{Re} & 1760 & 290 & 225 & 110 & 80 & 65
\end{array}
$$

TABLE 2. Channel Reynolds numbers $R e$ above which $R e_{p}>1$, for each particle size.

$R e$. The entry length is then given by $L_{e} / l \approx U / V_{\text {migr }}=6 \pi A^{-1} R e^{-1}(l / d)^{3}$. As shown in figure 14 , there is a decrease in $A$ when $R e$ is increased. This decrease suggests that the entry length will, in general, decrease slower than $R e^{-1}$, but nonetheless is predicted to decrease with increasing $R e$.

\section{Comparison and discussion}

\subsection{Segré-Silberberg equilibrium position}

In order to compare our results to the predictions of the asymptotic theory presented in the preceding section, we assume that the curvature in the pipe geometry has negligible influence: the channel width $l$ corresponds to the pipe diameter $D$, and the normalized distance from the channel centreplane $2 z / l$ corresponds to the normalized radius $r / R$. Figure 9 shows both the measured values of the equilibrium position, obtained at $L / D=310$, and the theoretical predictions as a function of $R e$. For the $D / d=42$ particles, it can be seen that the agreement is good when $R e<500$, even if, as on figure 8, migration is not completed for these particles at these $R e$. However, notice that the experimental values seem to be closer to the centre than those predicted by the theory when $R e>500$, although the difference is small. As particle size is increased, the discrepancy between asymptotic theory and experiments becomes larger. It appears that the asymptotic theory provides an upper bound for the equilibrium position. For instance, for the largest particles used, for which $D / d=8$, we observe an equilibrium position at $r=0.78$ for $R e=440$, whereas the predicted equilibrium position is at $r=0.87$.

Noting that the asymptotic theory assumes point particles, these discrepancies may be due to the two following finite-size effects:

(i) The theory assumes that the particle Reynolds number satisfies $R e_{p} \ll 1$, but this assumption is not valid for most of the particles investigated. Table 2 presents for each particle size the value of the pipe Reynolds number $R e$ above which $R e_{p}>1$. It can be seen that for the larger particles the assumption of small $R e_{p}$ is almost never valid in the range of $R e$ used. While the value of unity for $R e_{p}$ holds no particular meaning, a value of $R e_{p}=O(1)$ certainly invalidates the theory.

(ii) Another possible explanation may be the influence of the curvature of the pipe. The theoretical results were established in the case of a channel geometry, and it is possible that the curvature of the wall cannot be neglected when the particle radius is not small compared to that of the tube. We mention the curvature in connection with finite size of the particles, but the possibility exists that the force profile even for point particles may be qualitatively different in a cylindrical domain.

We note finally that there is an additional source of discrepancy which is probably more relevant in the case of the smaller particles: the asymptotic theory is inadequate in the case of an equilibrium position too close to the wall, i.e. for large $R e$. The outer region of the matched asymptotic theory corresponds to the region where advection balances viscous terms, and in the case of neutrally buoyant particles, the length scale of this region scales as $d R_{p}^{-1 / 2}$. The assumption that the flow around the particle can 


$\begin{array}{cccccc}D / d & 42 \pm 2.5 & 17 \pm 2.5 & 15 \pm 1.5 & 10.5 \pm 1 & 9 \pm 0.5 \\ L_{e} / D \text { at } R e=500 & 9500 & 620 & 430 & 150 & 92 \\ L_{e} / D \text { at } R e=1000 & 5600 & 370 & 260 & 90 & 55 \\ L_{e} / D \text { at } R e=1500 & 4650 & 310 & 210 & 73 & 46\end{array}$

TABLE 3. Pipe to particle diameter ratios and corresponding estimated entry lengths based on asymptotic theory. The pipe inner diameter is $D=8 \mathrm{~mm}$, and the scaled amplitudes of the lateral force $A(R e)$ were taken to be $A(500)=0.3, A(1000)=0.25$ and $A(1500)=0.2$. Measurements were performed at $L / D=310$.

be separated into an inner and an outer region is certainly invalid for these particles close to the wall.

\subsection{Entry length of the inertial migration}

Before discussing the entry length $L_{e}$ of the inertial migration, it is important to notice that the entry length $L_{P}$ of the Poiseuille flow for the pipe used in the experiment is of the order of $L_{P}=(\operatorname{Re} / 30) D \sim 50 \mathrm{~cm}$ for $R e=2000$ (Tritton 1988) and smaller for smaller Re. Therefore, the Poiseuille flow can be considered to be established in almost the entire length of the tube, and the influence of $L_{P}$ is neglected in the discussion of $L_{e}$.

We mentioned at the end of $\S 4$ that the entry length $L_{e}$ is predicted by the asymptotic theory to depend upon $R e$ and particle diameter $d$ as $L_{e} / D \approx 6 \pi A^{-1} R e^{-1}(D / d)^{3}$ (taking the conduit width, $l$, as the tube diameter, $D$ ). As indicated previously, $A$ decreases when $R e$ is increased. Hence, it is expected that $L_{e}$ decreases more slowly than $R e^{-1}$. The values of $L_{e}$ at three different Reynolds numbers have been computed for each particle size, and are shown on table 3. The typical magnitude of the scaled force has been taken as the local minimum of the force profiles in the region between the centre and the equilibrium position. The values of table 3 show that, except for the smallest particles, the entry length is of the order of (or smaller than) the tube length for all $R e>1000$.

It should be noted that the asymptotic theory predicts that the near-wall region develops much more rapidly, in fact, by more than an order of magnitude for $R e>100$, than the region at $r$ less than the Segré-Silberberg annulus because of the relative magnitudes of the lateral force in these two regions (see figure 14). This rapid near-wall variation of the particles' preferred radial location allows assessment of the location of the Segré-Silberberg annulus even when the entire distribution is not fully developed, in particular for the smallest particles.

In order to determine the evolution of the predicted particle distribution as a function of axial position for selected conditions, we performed a simple numerical integration of the equation of motion of a suspended particle, as discussed in $\S 4$. We calculate the positions at the location of the measurement (i.e. $L / D=310$ ) of an ensemble of independent particles initially randomly distributed over the crosssection; the particles are launched one by one into the tube. For $D / d=42$ and $R e=780$, starting from an initially random distribution at the entrance shown in figure 15(a), the predicted behaviour at our measurement location of $L / D=310$ seen on figure $15(b)$ is in qualitative agreement with the experimentally observed distribution of figure 8(a). However, the fraction of particles on the Segré-Silberberg annulus is larger in the simulation than in the experimental observation. Proceeding downstream in figures $15(c)$ and $15(d)$, the simulation prediction at $L / D=1250$ still 

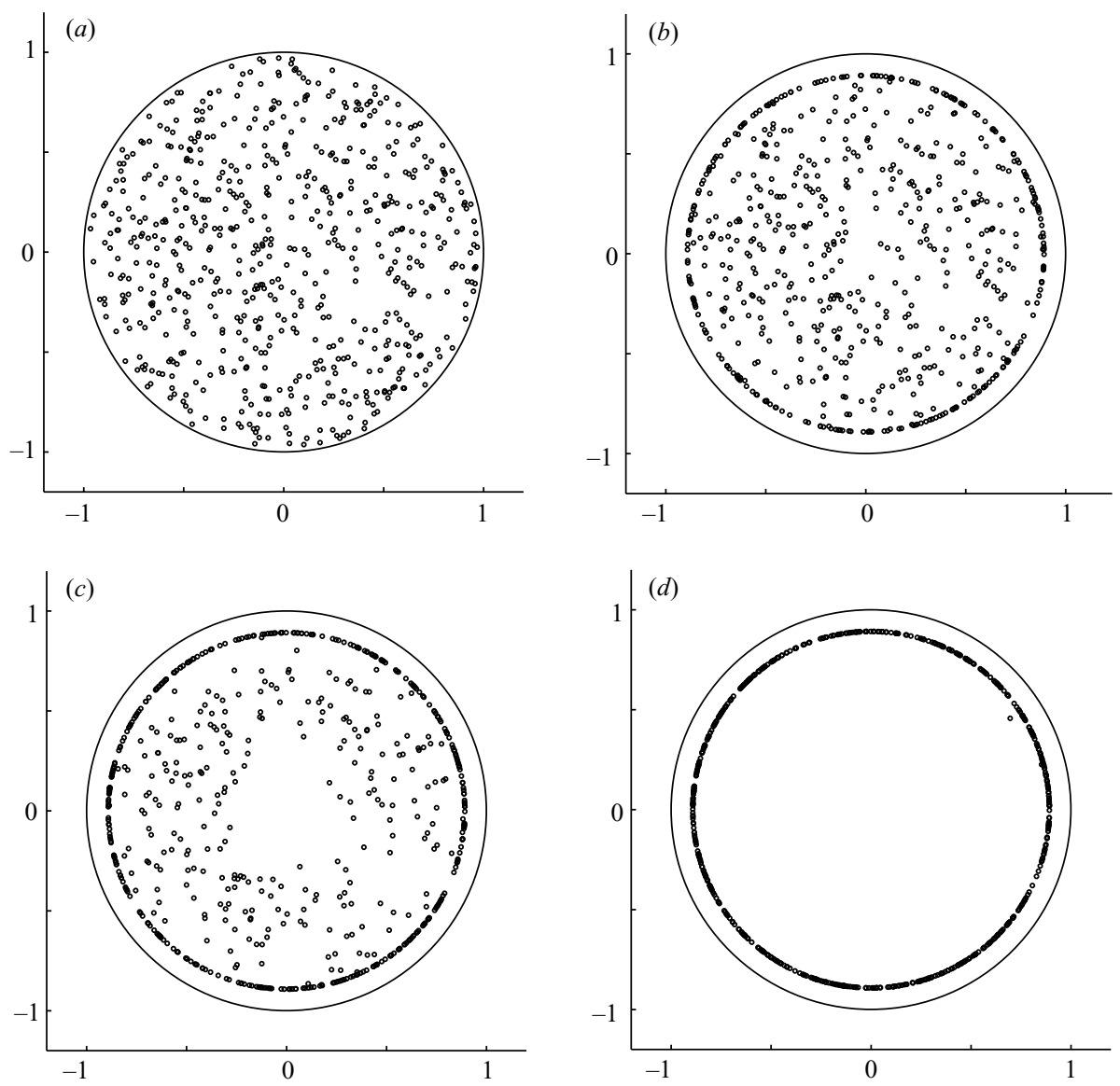

FIgURE 15. Predicted particle distributions over a cross-section for $D / d=42$ at $R e=780$ : (a) random distribution at the entrance, (b) $L / D=310$, (c) $L / D=1250$ and (d) $L / D=5000$.

shows an inner annulus, albeit containing fewer particles, which finally disappears at $L / D \approx 5000$. Conversely, for $D / d=9$ and $R e=350$, starting again from an initially random distribution at the entrance shown in figure $16(a)$, the predicted developed behaviour occurs well before the measurement location, as can be seen in figures 16(b)$16(d)$ which should be compared with the experimental results of figure 5 . Note in figure 16(a) that the initial random positions are displaced away from the wall because of the finite size of the particles.

\subsection{Inner annulus}

As mentioned in $\S 3$, the inner annulus, for example as seen in figure 6(a), was observed for $R e \geqslant 600$ for all particle sizes, except for the $D / d=42$ particles for which it was only observed above $R e=1200$. This particle distribution having an inner annulus seems at first to suggest that under these flow conditions there is a region of lower force for $r / R \sim 0.5$, and that particles consequently tend to accumulate in this inner region. This position coincides with the change in concavity of the force profile for large $R e$. For instance, it can be seen in figure 14 that at $R e=1000$, there is a broad weak minimum in radial force between two maxima of relatively larger force. 

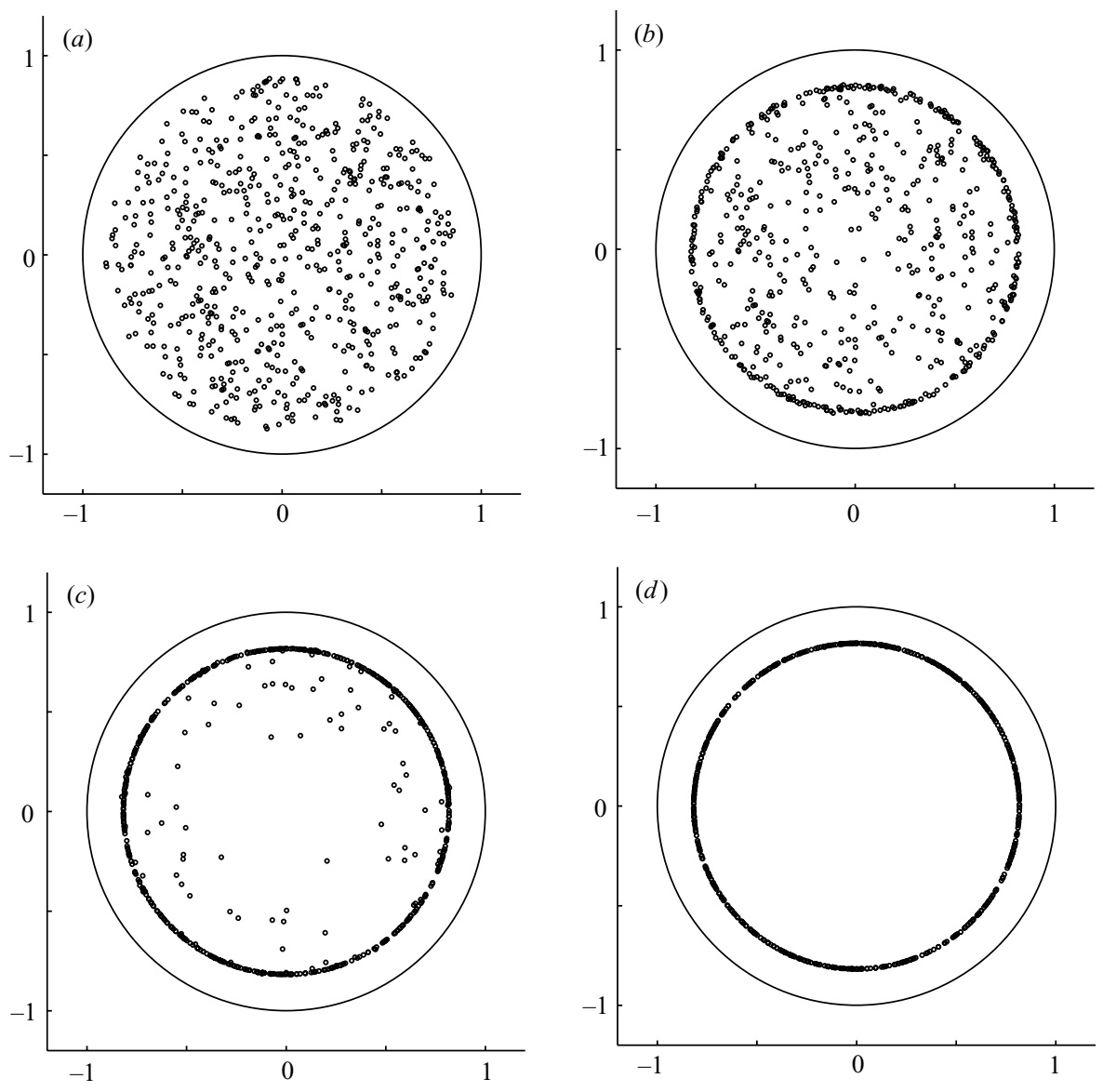

FIGURE 16. Predicted particle distributions over a cross-section for $D / d=9$ at $R e=350$ : (a) random distribution at the entrance, $(b) L / D=6,(c) L / D=25$ and $(d) L / D=50$. Note for comparison that the experimental measurement on figure 5 is at $L / D=310$.

Figure 17 shows that the position of this region of weak force seems to agree with the observed inner maximum in the experimental radial distribution. The question is whether this inner annulus is actually a real equilibrium position, i.e. a zero of the force profile not captured by the theory for channel flow, or just the result of a slower migration around $r / R=0.5$. The asymptotic theory predicts that the inner annulus will disappear if the pipe is long enough to allow the system to reach a fully developed state.

To illustrate this last point and to determine whether the inner annulus is a transient, we can compare the predictions of the simulation of particle trajectories in the preceding section with the experimental observations. It can be seen in figures 15 and 16 that an inner annulus can be distinguished in the result of the simulation, but that it differs qualitatively from that experimentally observed. The proportion of particles on the outer annulus is much larger in the simulation, and the radius of the inner annulus appears to be larger in the simulation. More generally, the simulation based on the theory predicts that the axial location of the inner annulus, i.e. the place in the pipe where this transient feature is observed, will strongly depend on particle size as $L \sim(D / d)^{3}$. However, as shown in figure 7 , the particle distributions 


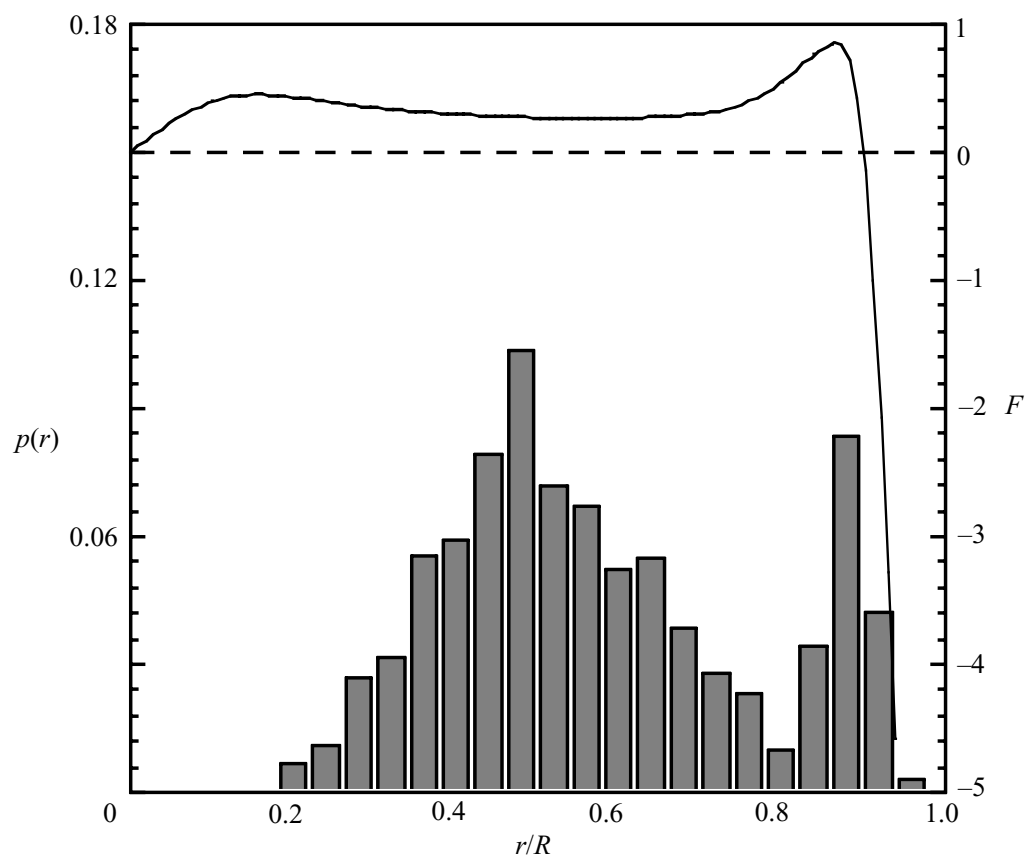

FIGURE 17. Histogram of radial positions for $D / d=17$ and $R e=1000$, and scaled radial force profile for this Reynolds number $F=F_{l} R_{c}^{1 / 2} \epsilon^{-3} /\left(\eta U_{m} a\right)$.

on the inner and outer annulus are essentially independent of particle size: at a given $R e$ there is the same fraction of particles on the inner annulus for large or small particles. This strongly suggests that the inner annulus is not a transient feature.

In addition to demonstrating that the measured particle distributions depend little on particle size over the range of $450-900 \mu \mathrm{m}$ particles, figure 7 shows that when $R e$ is increased there is a major shift from the outer annulus to the inner annulus. Figure 6 also illustrates this trend, and shows the experimental variation of the inner annulus as $R e$ increases from $R e=1000$ in figure $6(a)$ for $D / d=17$. If this inner annulus were a transient, it would be expected to disappear at large $R e$, owing to the expected decrease of $L_{e}$ with $R e$. Because it is, on the contrary, enhanced for large $R e$ is another argument in favour of the inner annulus being a real equilibrium position.

We can finally observe that figures $6(b)$ and $6(d)$ show situations where very few particles are present on the outer annulus: these distributions suggest that particles initially present in the outer region at the entrance of the pipe actually leave that region to reach the inner annulus. This is another argument pointing to the stability of the inner annulus. These experimental distributions even suggest that at $R e \approx 1600$ the inner annulus could be the only equilibrium position in the pipe. We thus conclude that experimental observations suggest the inner annulus is a real equilibrium position rather than a transient feature. The failure of the asymptotic theory in predicting such a distribution could be due to the point-particle assumption, the difference in geometry in the theory and experiment, or a combination of these factors. Taking into account either particle size or the cylindrical geometry is expected to induce changes in the force profiles computed for plane Poiseuille flow, although the relevance to our observations awaits such calculations. We note that the finite size of the particles 

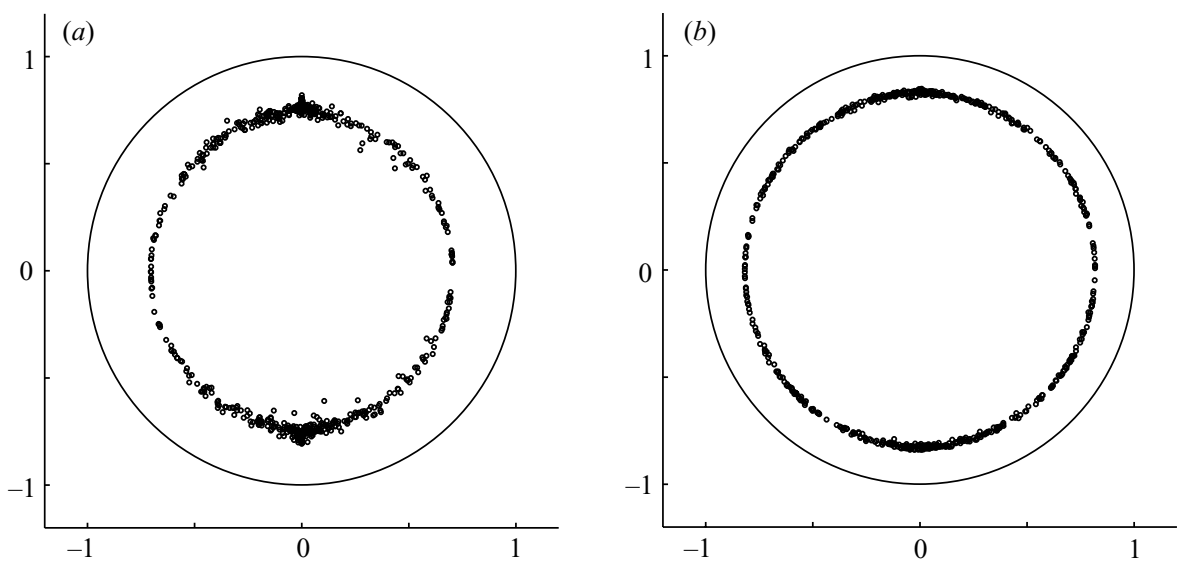

FiguRE 18. Predicted particle distributions over a cross-section for $D / d=15$ :

(a) $B_{\sigma}= \pm 0.0036$ and $R e=170,(b) B_{\sigma}= \pm 0.0016$ and $R e=390$.

results in finite-particle Reynolds numbers $R e_{p}$ involved in the experiment, and except for the smallest particles, all of the results in figure 7 are at $R e_{p}>1$.

\subsection{Slightly non-neutrally buoyant particles}

We have presented in $\S 3$ measurements of the particle distribution when inertial migration is accompanied by buoyancy effects for a set of particles presenting a dispersion in density about a mean density equal to that of the fluid, with results in figure 11 for $D / d=15$. We estimated the dispersion to be a Gaussian with a standard deviation of $0.001 \mathrm{~g} \mathrm{~cm}^{-3}$ about the mean; at one standard deviation $B_{\sigma}= \pm 0.038$ for $R e=170$, and $B_{\sigma}= \pm 0.017$ for $R e=390$. The predictions of the simulation for the same conditions as figure 11 are presented in figure 18. The agreement is good as the accumulation of particles at the poles is pronounced at $R e=170$, but essentially disappears at $R e=390$. We note, however, that the Segré-Silberberg annulus is more stretched in the vertical direction than is actually observed in the experiments.

We have also examined in $\S 3$ the migration patterns in the presence of a weak sedimentation for particles with a mean density slightly greater than the fluid; see figure 12. The predictions of the simulation for the conditions of figure 12(b), i.e. $B=0.025, R e=650$ and $D / d=15$, are shown in figure 19 . We used here a small Gaussian dispersion about the mean density as described in the preceding paragraph, and considered the same number of particles as were sampled in the experiments to make the comparison easier. Starting from a random distribution at the entrance of the tube on figure 19(a), the hole in the middle of the distribution opens at $L / D \approx 80$ and the simulated distribution presents an obvious asymmetry caused by settling (see figure 19b). We find a good agreement with the experimental observation of figure $12(b)$ at the measurement location $L / D=310$, with simulation results presented in figure $19(c)$. The inner annulus can still be clearly distinguished on the simulated cross-section, though its lower part is devoid of particles, as both settling and inertial migration move the particles toward the lower wall in this region. The Segré-Silberberg annulus is clearly visible in the lower part, but is depopulated in its upper part. When the simulation is run further, the particles tend to accumulate at the bottom, as illustrated in figure $19(d)$ for $L / D=625$ and will eventually converge into a single point. The presentation of results in the latter part of the evolution, i.e. 

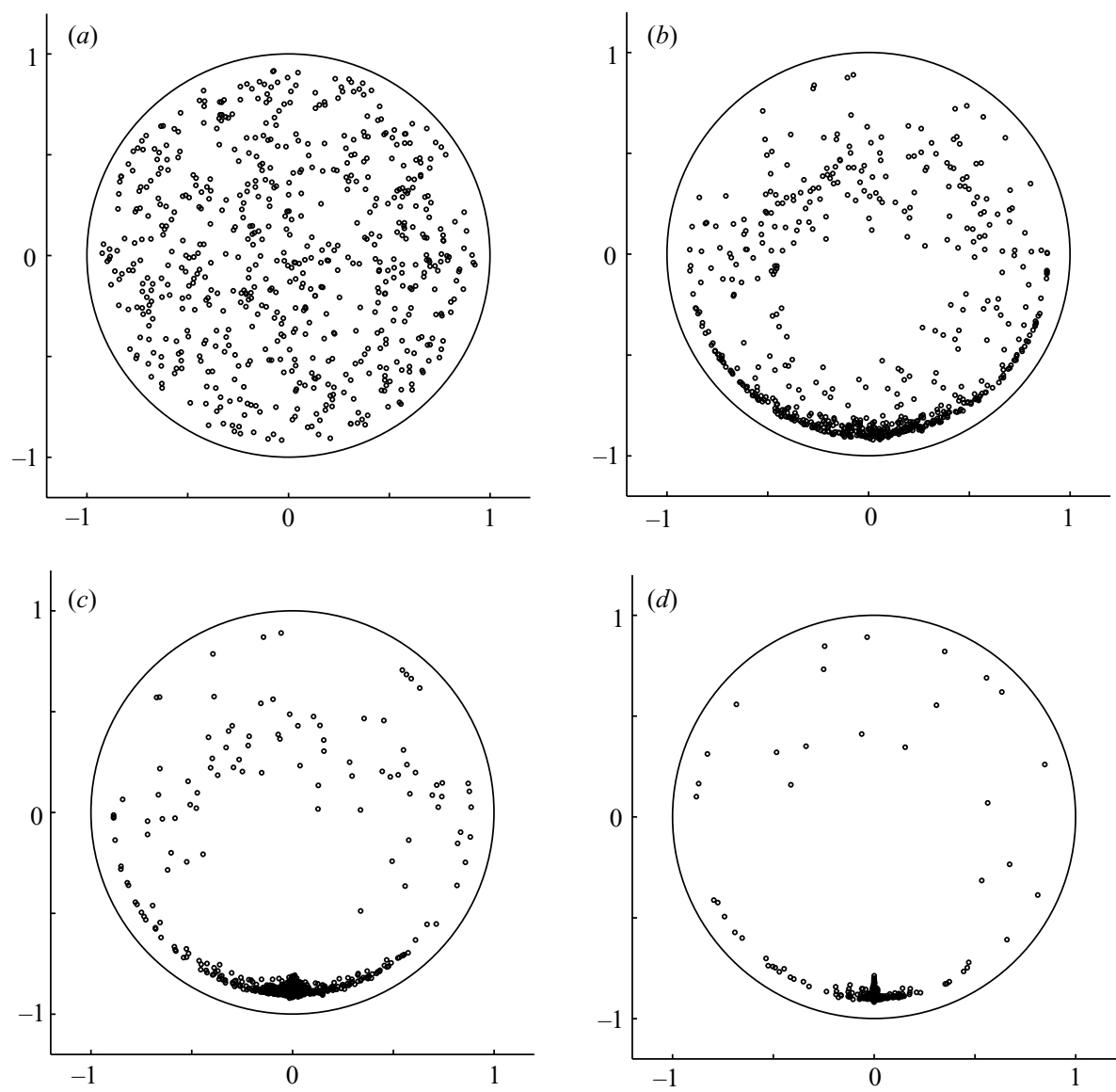

FiguRE 19. Predicted particle distributions over a cross-section for $D / d=15, R e=650$ and $B=0.0025:$ (a) Initial random distribution, (b) $L / D=125$, (c) $L / D=310$ and $(d) L / D=625$. Note that the theory predicts convergence to a single point as $L / D \rightarrow \infty$; the number of particles considered is the same in each case (630).

in figure $19(c, d)$, for the simulation may be somewhat misleading unless it is noted that the points become localized on small portions of the cross-section and become indistinguishable. While it thus appears fewer particles have been sampled, this is not the case. We should mention that in spite of the qualitative agreement of the theory and experiment for the sedimenting particles, the criterion of Hogg (1994), noted in $\S 4, B(d / 2 D)^{-2} \ll 1$ does not hold in these conditions. We have $(B / 2)(d / 2 D)^{-2}=1$, meaning that the Stokeslet due to particle sedimentation is not negligible near the wall. The second condition on the inertial length scales of sedimentation and shear flow $R_{c}(B / 2)^{2} \ll 1$ is met with $R_{c}(B / 2)^{2}=10^{-3}$.

\section{Conclusion}

In this work we have examined the influence of inertia on the radial migration of rigid spherical particles in Poiseuille flow. We have extended the work of Segre \& Silberberg (1962) for neutrally buoyant particles up to $R e \approx 2000$. Our results show 
that the equilibrium radial position for this case, which we have termed the SegreSilberberg annulus, moves toward the wall as $R e$ is increased. Our data for large particles, meaning small $D / d$, are in agreement with those of the Segré \& Silberberg experiments performed for particles of similar $D / d$. The results for small particles, $D / d=42$, are in good agreement with the predictions of asymptotic theory (Ho \& Leal 1974; Schonberg \& Hinch 1989; Hogg 1994; Asmolov 1999) up to $R e \approx 500$. The results for large particles do not agree with the asymptotic theory in the following sense: the experimental equilibrium position is moved toward the centre of the pipe relative to the predictions of this theory. This deviation from theory increases as $D / d$ becomes smaller (increasing size of particles for fixed tube diameter). The discrepancy can be explained as a particle size effect. The theory is strictly valid only for $\operatorname{Re}_{p} \ll 1$, a condition not met in our experiments. In addition, the theory is developed for a channel flow, and the curvature of the pipe in the experiments cannot be neglected when $D / d$ is too small. A further source of discrepancy is that the theory may break down for an equilibrium position too close to the wall.

In these experiments, we have observed an accumulation of particles on an inner annulus, i.e. at smaller radial position than the Segré-Silberberg annulus. This occurs for $R e>600$ for all particles except the smallest set, for which this was observed above $R e=1200$. This inner annulus coincides with the change in concavity of the force profile predicted by asymptotic theory for channel flow at large $R e$. In this work, we questioned whether this annulus corresponds to a true zero of the force or is a transient feature, observed only when the entry length is longer than the system. Since experiments were performed at a single downstream position, we did not study the evolution of particle distributions with position to a final steady state. However, the following points are another way of arguing in favour of the stability of the inner annulus.

The entry length for migration depends, according to theory as described in $\S 5.2$, upon the tube (channel in the theory; Asmolov 1999) Reynolds number and the ratio of tube to particle size, $D / d$. The entry length is predicted to decrease as $R e$ increases - more slowly than $R e^{-1}$, but nonetheless to decrease. Focusing first on large particles (small $D / d$ ) and taking a specific condition, we observe that for sufficiently small Re particles of $D / d=9$ are fully migrated to the Segré-Silberberg equilibrium, as seen in figure 4 at $R e=67$. Thus we must expect that for larger $R e$, these same particles will have completed their migration by the same measurement station, and if not, the theory is incorrect in the scaling with $R e$. What we observe is that with increasing $R e$ there is a smooth transition from accumulation on the theoretically predicted equilibrium position to the inner annulus as indicated by figure 7 , and hence the results show some form of breakdown of the theory. Opening consideration to include particle size, the essential independence of this transition with $\operatorname{Re}$ from $D / d$, combined with the strong dependence of the entry length upon this ratio predicted by the theory as noted in $\S 5.2$, points to the inner annulus as an equilibrium location rather than a transient. We note that this new equilibrium position would require only a change in the detailed form of the force profile with respect to radial position from that predicted by the theory for channel flow. In sharp contrast, the inner annulus as a transient feature, with the probability of distribution to this and the SegreSilberberg position independent of particle size, would require a complete breakdown of the existing theory with regard to both $R e$ and particle size. While it is difficult to envisage such a breakdown in scaling, there remains significant uncertainty that could be resolved by further experiments and numerical analysis, with a study of the theory for the cylindrical geometry a natural starting point in the latter case. 
The influence of concentration on lateral migration was not investigated in this study. However, Han et al. (1999) observed that concentration effects do not modify the location of the Segré-Silberberg annulus, but only induce a strong dispersion in the particle distribution. We observed experimentally that when particle interactions were not negligible, particles present at the Segré-Silberberg annulus tended to become structured in alignments parallel to the axis of the flow, an effect already pointed out as 'chain' formation by Segré \& Silberberg (1962). This effect, which is studied in Matas et al. (2004), was mostly avoided in the present investigation by keeping the volume fraction low enough to limit interactions between particles.

We have investigated the influence of buoyancy in two cases, one being a slight dispersion of particle density about that of the fluid, and the second for a set of particles all slightly heavier than the fluid. For the case of a dispersion in density, we observe an accumulation at the top and bottom of the cross-section. This accumulation disappears as the ratio of inertia to buoyancy is increased through an increase of the Reynolds number. In the second case, the inertial migration competes with settling above the tube centreline while the two act in concert below, and this results in a strong asymmetry of the distribution. In both cases, simulation of the particle trajectories based on asymptotic theory captures the general features observed in the experiments.

The authors thank F. Ratouchniak for technical assistance with the apparatus. The work was supported in part by the Institut Français du Pétrole, and was undertaken under the auspices of a CNRS-NSF cooperative research project 'Flow, resuspension, and sedimentation of a suspension in a tube', (NSF award 0129079 and CNRS award 12940). A Fellowship from the French Ministère de la Recherche is gratefully acknowledged by J. P. M. The hospitality of Laboratoire IUSTI during the preparation of this work is gratefully acknowledged by J. F. M.

\section{REFERENCES}

Aoki, H., Kurosaki, Y. \& AnZaI, H. 1979 Study on the tubular pinch effect in a pipe flow. Bull. JSME 22 164, 206-212.

Asmolov, E. S. 1999 The inertial lift on a spherical particle in a plane Poiseuille flow at large channel Reynolds number. J. Fluid Mech. 381, 63-87.

Bretherton, F. P. 1962 The motion of rigid particles in a shear flow at low Reynolds number. J. Fluid Mech. 14, 284-304.

Han, M., Kim, C., Kim, M. \& LeE, S. 1999 Particle migration in tube flow of suspensions. J. Rheol. 43, 1157-1174.

Ho, B. P. \& LEAL, L. G. 1974 Inertial migration of rigid spheres in two-dimensional unidirectional flows. J. Fluid Mech. 65, 365-400.

HogG, A. J. 1994 The inertial migration of non-neutrally buoyant spherical particles in twodimensional shear flows. J. Fluid Mech. 272, 285-318.

Jefrerey, R. C. \& Pearson, J. R. A. 1965 Particle motion in laminar vertical tube flow. J. Fluid Mech. 22, 721-735.

Karnis, A., Goldsmith, H. L. \& Mason, S. G. 1966 The flow of suspensions though tubes. V Inertial effects, Can. J. Chem. Engng 44, 181-193.

Matas, J.-P., Glezer, V., Morris, J. F. \& Guazzelli, É. 2004 Trains of particles in finite-Reynoldsnumber pipe flow. Phys. Fluids (submitted).

Matas, J.-P., Morris, J. F. \& Guazzelli, É. 2003 Transition to turbulence in particulate pipe flow. Phys. Rev. Lett. 90, 014501.

Oliver, R. 1962 Influence of particle rotation on radial migration in the Poiseuille flow of suspensions. Nature 194, 1269-1271. 
RePetti, R. V. \& LeONARD, E. F. 1964 Segré-Silberberg's annulus formation: a possible explanation. Nature 203, 1346-1348.

Rubinow, S. I. \& Keller, J. B. 1961 The transverse force on a spinning sphere moving in a viscous fluid. J. Fluid Mech. 11, 447-459.

Saffman, P. G. 1965 The lift on a small sphere in a slow shear flow. J. Fluid Mech. 22, 385-400.

Schonberg, J. A. \& Hinch, E. J. 1989 Inertial migration of a sphere in Poiseuille flow. J. Fluid Mech 203, 517-524.

Segré, G. \& Silberberg, A. 1962 Behaviour of macroscopic rigid spheres in Poiseuille flow Part 2. Experimental results and interpretation. J. Fluid Mech. 14, 136-157.

TaChibana, M. 1973 On the behaviour of a sphere in the laminar tube flows. Rheol. Acta 12, 58-69.

Tritton, D. J. 1988 Physical Fluid Dynamics, 2nd edn. Oxford Science. 